\title{
Biological and small molecule strategies in migraine therapy with relation to the CGRP family of peptides.
}

\author{
Lars Edvinsson (GUEST EDITOR) ${ }^{1}$, Jacob Edvinsson², and Kristian Agmund Haanes ${ }^{3}$ \\ ${ }^{1}$ Institute of Clinical Science in Lund, Lund University \\ ${ }^{2}$ University of Copenhagen Faculty of Health and Medical Sciences \\ ${ }^{3}$ Copenhagen University Hospital, Glostrup
}

April 3, 2021

\author{
Abstract \\ Migraine is one of the most common of neurological disorders with a global prevalence of up to $15 \%$. One in five migraineurs \\ have frequent episodic or chronic migraine requiring prophylactic treatment. In recent years, specific pharmaceutical treatments \\ targeting calcitonin gene-related peptide (CGRP) signalling molecules have provided safe and effective treatments; monoclonal \\ antibodies for prophylaxis and gepants for acute therapy. Albeit the beneficious impact of these new drugs, it is important to \\ understand the molecular mechanisms involved to better understand migraine pathophysiology and improve the therapy. Here \\ we describe current views on the role of the CGRP family of peptides CGRP, calcitonin (CT), adrenomedullin (AM), amylin \\ (AMY) and their receptors in the trigeminovascular system (TGV). All these molecules are present within the TGV system \\ but differ in expression and localization. It is likely that they have different roles, which can be utilized in providing additional \\ drug targets. \\ Review Paper for Br. J. Pharmacol. 2021. \\ Biological and small molecule strategies in migraine therapy with relation to the CGRP family \\ of peptides. \\ Lars Edvinsson ${ }^{1,2}$, Jacob C. A. Edvinsson ${ }^{2,3}$, Kristian A. Haanes ${ }^{1,2}$ \\ ${ }^{1}$ Departments of Internal Medicine, Lund University Hospital, Lund, Sweden. \\ ${ }^{2}$ Department of Clinical Experimental Research, Glostrup Research Institute, Glostrup Hospital, Rigshospi- \\ talet, Denmark. \\ ${ }^{3}$ Department of Drug Design and Pharmacology, Faculty of Health and Medical Sciences, University of \\ Copenhagen, Copenhagen, Denmark. \\ Address for correspondence: \\ Lars Edvinsson, Professor MD \\ Department of Internal Medicine, Lund University Hospital, S-22185 Lund, Sweden. \\ Phone: +46703271484 \\ e-mail: lars.edvinsson@med.lu.se
}




\section{Abstract}

Migraine is one of the most common of neurological disorders with a global prevalence of up to $15 \%$. One in five migraineurs have frequent episodic or chronic migraine requiring prophylactic treatment. In recent years, specific pharmaceutical treatments targeting calcitonin gene-related peptide (CGRP) signalling molecules have provided safe and effective treatments; monoclonal antibodies for prophylaxis and gepants for acute therapy.

Albeit the beneficious impact of these new drugs, it is important to understand the molecular mechanisms involved to better understand migraine pathophysiology and improve the therapy. Here we describe current views on the role of the CGRP family of peptides CGRP, calcitonin (CT), adrenomedullin (AM), amylin (AMY) and their receptors in the trigeminovascular system (TGV). All these molecules are present within the TGV system but differ in expression and localization. It is likely that they have different roles, which can be utilized in providing additional drug targets.

Key words . CGRP, adrenomedullin, amylin, calcitonin, CLR, RAMPs, receptors

\section{INTRODUCTION}

Migraine is a complex disorder, known for centuries but still not fully understood. Affecting 1 billion people, migraine is one of the most prevalent, disabling neurological disorders worldwide. Migraine has a female predominance with debilitating impact in the most active years (2018; GBD 2016 Disease and Injury Incidence and Prevalence Collaborators, 2017). Currently, the migraine attack is proposed to start in the central nervous system (CNS), mainly involving regions such as hypothalamus and brainstem (Goadsby, Holland, Martins-Oliveira, Hoffmann, Schankin \& Akerman, 2017). Evidence of migraine initiating in the CNS has been demonstrated in longitudinal neuroimaging studies over 30 days in spontaneous migraine attacks (Schulte, Mehnert \& May, 2020; Schulte, Menz, Haaker \& May, 2020). The studies revealed that hypothalamic activation during the premonitory phase of a spontaneous migraine attack, and ictally there was activity in brainstem regions often discussed in migraine. However, the link with the trigeminovascular (TGV) system and the CNS is still unclear; either the CNS is the sole activator of the trigeminal nucleus caudalis (TNC) and subsequently the TGV system or the incoming signals from the TGV system can be modified by brainstem nuclei. Following activation of the $1^{\text {st }}$ order neurons in the TG, the sensory nerve fibres transmit pain signals to the lower brainstem and spinal cord C1 - C3, verified in tracing studies, and send signals to second order ascending neurons to various CNS regions such as brainstem nuclei and thalamus (Edvinsson, 2011).

Until 25 years ago, neurologists had few, and often insufficient, options to treat patients with acute migraine attacks apart from general analgesics alone or in combinations. Understanding the mechanisms of ergotamine related molecules resulted in development of the triptan group of acute medications; acting with high specificity on serotonin $\left(5-\mathrm{HT}_{1 \mathrm{~B}}\right.$ and $\left.5-\mathrm{HT}_{1 \mathrm{D}}\right)$ receptors. The triptans may act via at least 3 sites; (i) they are cranial vasoconstrictors with possibility of cardiovascular side-effects (Saxena \& Den Boer, 1991), (ii) they inhibit neuropeptide release (Amrutkar, Ploug, Hay-Schmidt, Porreca, Olesen \& Jansen-Olesen, 2012; Goadsby \& Edvinsson, 1994), and (iii) may inhibit second-order neurons of the TGV pain pathway (Goadsby \& Knight, 1997).

Due to the high probability of cardiovascular adverse events, there was a need for new drugs without vasoconstrictor effects. This work lead to a new group of prophylactic agents, monoclonal antibodies (mAbs) towards CGRP or selective binding to CGRP receptor components. These new mAbs have since proven to be effective and have few side-effects (Edvinsson, Haanes, Warfvinge \& Krause, 2018). Currently, upcoming additions to acute anti-migraine pharmacotherapy includes the $5-\mathrm{HT}_{1 \mathrm{~F}}$ receptor class of agonists, the ditans (Labastida-Ramirez et al., 2020), and the CGRP receptor antagonists, the gepants (Edvinsson, Haanes, Warfvinge \& Krause, 2018).

This review provides a brief description of mechanistic actions of the molecules acting through CGRP related mechanisms in relation to migraine therapy, and discusses the actions based on available molecular data. 
Combined, we propose ways towards improving therapy of acute and prophylactic treatments of migraine at sites involving other members of the CGRP family of peptides.

\section{THE CGRP SYSTEM and MIGRAINE.}

CGRP and its receptor are extensively expressed in two separate neuron populations in the TGV system (Eftekhari, Salvatore, Calamari, Kane, Tajti \& Edvinsson, 2010). CGRP is released from the TGV system in conjunction of acute attacks of migraine and cluster headache (Ho, Edvinsson \& Goadsby, 2010). The trigeminal C-fibres and ganglia are the main source of stimulus-induced CGRP found in the jugular vein as shown in man and cat (Goadsby, Edvinsson \& Ekman, 1988) and rat (Hoffmann, Wecker, Neeb, Dirnagl \& Reuter, 2012). The C-fibres have both afferent and efferent aspects; perivascular fibres release CGRP onto the vascular smooth muscle cells to induce dilatation and on dural mast cells to participate in induction of neurogenic inflammation (Pietrobon \& Moskowitz, 2013). Many outstanding questions remain, particularly regarding the other members of the CGRP family. This group of signalling peptides include calcitonin (CT), adrenomedullin (AM) and amylin (AMY) and their receptors (Table 1). A fair question is often asked: Can other peptides be released in the TGV and will CGRP acting drugs have effects that include other related receptor targets?

\section{CGRP family; peptides and receptors.}

The CGRP family of peptides ( $\alpha$ and $\beta$ forms) are ligands for a closely related family of $G$ protein-coupled receptors (GPCRs) with shared structural homology (Hay et al., 2014). The CGRP family of peptides and receptors show a varying degree of structural homology with CGRP and have a widespread distribution throughout the body (Hendrikse, Bower, Hay \& Walker, 2018).

The receptors in this group are formed from either of two 7-transmembrane (TM) G-protein coupled receptors (GPCRs), the calcitonin receptor-like receptor (CLR) or the calcitonin receptor (CTR), which interact with receptor activity-modifying protein (RAMPs) to form heterodimers (Gingell, Hendrikse \& Hay, 2019). The RAMPs are a small family of three proteins (RAMP 1-3) that are single TM-spanning proteins that can modify binding characteristics, pharmacology, functionality and cell trafficking of the specific GPCRs (Hay \& Pioszak, 2016). The currently most central is the 7-TM complex - CLR - which is a required element of receptors for CGRP and adrenomedullin $\left(\mathrm{AM}_{1}\right.$ and $\left.\mathrm{AM}_{2}\right)$. Early studies showed that transfecting cells with only CLR revealed no response to CGRP (McLatchie et al., 1998). It was only after the demonstration that the dimerization of RAMP1 and CLR resulted in formation of a functional receptor to CGRP (Hay, Garelja, Poyner \& Walker, 2018). When CLR couples to RAMP2 or RAMP3 it forms AM $_{1}$ receptor (CLR/RAMP2) or $\mathrm{AM}_{2}$ receptor (CLR/RAMP3). The AMY receptors are formed by association of the Calcitonin (CT) receptor (CTR) with a RAMP: $\mathrm{AMY}_{1}$ receptor (CTR/RAMP1), $\mathrm{AMY}_{3}$ receptor (CTR/RAMP3). The CTR can also signal on its own, as a calcitonin receptor (without any RAMP) (Poyner et al., 2002). Due to the complexity of this peptide-receptor system, their expression in the trigeminal system is unclear and their functional roles yet to be resolved (Edvinsson, Grell \& Warfvinge, 2020).

\section{Localization of the CGRP family of peptides in relation to the TGV system.}

CGRP is expressed in a granular pattern in small $(<30 \mu \mathrm{m})$ to medium $(30-60 \mu \mathrm{m})$ sized TG neurons (Eftekhari, Salvatore, Calamari, Kane, Tajti \& Edvinsson, 2010; Lennerz et al., 2008) where CGRP is packed in vesicles that are surrounded by the Golgi apparatus. In addition, C-type of sensory unmyelinated nerves appear as pearl-like CGRP immunoreactivity (ir) in boutons (also known as varicosities) (Figure 1). The myelinated fibres do not contain CGRP as sometimes has been proposed (Eftekhari, Warfvinge, Blixt \& Edvinsson, 2013; Lennerz et al., 2008). The CGRP receptor components, CLR and RAMP1, are co-expressed in medium to large $(>60 \mu \mathrm{m})$ diameter neurons (Eftekhari et al., 2016; Eftekhari, Salvatore, Calamari, Kane, Tajti \& Edvinsson, 2010). In addition, CLR and RAMP1 have been observed in satellite glial cells (SGC) and in thinly myelinated fibres, typical for Aס-fibres in various parts of the TGV system such as the TG, dura mater, and root entry zone (Figure 1) (Eftekhari, Salvatore, Calamari, Kane, Tajti \& Edvinsson, 2010; Lennerz et al., 2008; Miller et al., 2016). Early findings, which have today been confirmed by many researchers, has stood the test of time, and served a solid base for the successful development of 
mAbs and small molecule gepants (Edvinsson, Haanes, Warfvinge \& Krause, 2018).

$C T$ is a hormone produced mainly by $\mathrm{C}$ cells in the thyroid gland with a role to reduce plasma calcium and to promote bone formation (Findlay \& Sexton, 2004). In the clinic, CT is used in treatment of bone disorders characterized by increased bone resorption, osteoporosis and hypercalcemia due to malignancy, with some pain relief (Findlay \& Sexton, 2004). Thus, CT and its receptor CTR are seen in different cell types and tissues which suggests multiple physiological roles, including bone metabolism (Findlay \& Sexton, 2004). CT has not been shown to be expressed in the nervous system, however binding sites for CT are found in many brain structures (Hendrikse, Bower, Hay \& Walker, 2018). In addition, a recent study has revealed the presence of the CT receptor in the human brainstem (Bower et al., 2016).

Recently, CT was found to be expressed in the trigeminal system; CT the peptide was found in vesicles that are surrounded by the endoplasmic reticulum/Golgi apparatus and occur in addition as pearl-like CTimmunoreactivity (ir) in the C-type of sensory fibres (Figure 1B) (Edvinsson, Grell \& Warfvinge, 2020).

. The CT-ir was expressed in a pattern like that of CGRP; granular staining of small to medium sized neurons and pearl-like staining of fibres in the TG. In addition, many SGCs were CT positive (Edvinsson, Grell \& Warfvinge, 2020). In a subpopulation of TG neurons there is granular and cytosolic expression of CT-ir (Figure 1).

AMY is currently in focus of much migraine research, due to its affinity for the CGRP receptor that pharmacologically is almost equal to that of CGRP and because CGRP has strong affinity for the AMY 1 receptor (Hendrikse, Bower, Hay \& Walker, 2018). Comparing the binding affinity of olcegepant and $\mathrm{CGRP}_{8-37}$ to the $\mathrm{AMY}_{1}$ and CGRP receptors revealed similarities in binding characteristics as well as in cAMP production (Walker et al., 2015). Human AMY was first isolated in 1987 (Cooper, Willis, Clark, Turner, Sim \& Reid, 1987). It is an endocrine hormone that signals to the brain and acts as a satiety factor; its presence in plasma is about 10 times higher than that of CGRP (Hay, 2017). Research on AMY deposition in brain neurons has been discussed for a role in Alzheimer's disease (Mietlicki-Baase et al., 2017) and AMY has been found to alter viability of human brain pericytes (Schultz, Byman, Fex \& Wennstrom, 2017).

Previously, we reported that AMY is expressed in feline TG neurons, and that AMY relax in vitro and in vivo cerebral vessels (Edvinsson, Goadsby \& Uddman, 2001). A detailed report showed AMY expression in a few small to medium sized TG neurons which co-express CGRP and in C-fibres (Edvinsson, Grell \& Warfvinge, 2020).

Receptors for CGRP and AMY are related and share components, CLR/RAMP1 and CTR/RAMP1, respectively. Given the close relationship between AMY and CGRP, and since release of the peptides may act on either or both receptors, it is understandable that experiments with AMY aimed to trigger migraine and to block migraine with mAbs towards AMY are on the way. However, early result from a trial on migraine patients did not reveal any prophylactic effect of the mAbs towards AMY (Ashina, 2019).

AM is most often considered an endothelial peptide and was first isolated in 1993 (Kitamura et al., 1993). AM is generally expressed and participates in a variety of physiological functions including vasodilation, bronchodilatation, growth and hormone regulation (Ferrero et al., 2018). Furthermore, AM is involved in pathophysiological processes such as hypertension, retinopathy and tumour genesis (Ferrero, Larrayoz, GilBea, Martinez \& Ramirez, 2018). In mammals, endothelial AM-ir is present in low concentrations in the vascular endothelium (Satoh et al., 1996). AM is found in neurons and glial cells (Serrano et al., 2000). We have reported the presence of AM-ir in the thin cytoplasm of the glial cells, SGCs and cells enveloping neuronal processes, probably myelinating cells. In addition, AM-ir has been noted to occur in cranial vascular endothelium (Edvinsson, Grell \& Warfvinge, 2020). Its molecular relation to CGRP has resulted in suggestion that AM may have a role in migraine pathophysiology (Juhl, Petersen, Larsen, Jansen-Olesen \& Olesen, 2006). However, intravenous AM infusion did not cause migraine-like attacks in man (Ashina, Hansen, BO \& Olesen, 2017). This view is in line with our demonstration that there is no AM in TG neurons but presence in glial cells and vascular endothelium (Edvinsson, Grell \& Warfvinge, 2020). 


\section{Relation between expression of the peptides and their receptors.}

CGRP and CT were found to be co-expressed in small to medium sized neurons, and in some SGCs, suggesting that CT might have a role in trigeminal function (Edvinsson, Grell \& Warfvinge, 2020). CGRP and AMY are co-expressed in some of the small to medium sized neurons and in thin C-fibres. The number of CGRP positive cells were in abundance compared to those storing AMY (Edvinsson, Grell \& Warfvinge, 2020). AMY is present in high concentrations in plasma, in migraine attacks higher levels of CGRP is released than AMY. Since the CGRP receptor is more sensitive to CGRP than to AMY, AMY is unlikely to have a central role in migraine.

The CGRP receptor (CLR/RAMP1) is mainly expressed in large neurons and SGCs (Edvinsson, Grell \& Warfvinge, 2020). The $\mathrm{AM}_{2}$ receptor (CLR/RAMP3) occurs in some SGCs, the AMY receptor (CTR/RAMP1) in large neurons and SGCs, while the $\mathrm{AMY}_{3}$ receptor (CTR/RAMP3) has been observed in some SGCs.

\section{SITE OF ACTION OF CGRP AND THE CGRP RECEPTOR ACTIVE DRUGS.}

CGRP antagonistic molecules have by now a 2-decade history with olcegepant and telcagepant as the first small molecules that competitively antagonize the vasodilator effect of CGRP in human arteries (Edvinsson et al., 2002). They were studied in several clinical trials, but the program was halted because of hepatotoxicity. Following molecular modifications three other gepants have now passed phase III and are approved by the FDA; these are atogepant, ubrogepant (Rubio-Beltran, Chan, Danser, MaassenVanDenBrink \& Edvinsson, 2020) and rimegepant (Mulder et al., 2020). The drugs were initially designed for acute therapy, but they are now also studied for prophylaxis. The other class of molecules are humanized monoclonal antibodies directed towards different parts of the CGRP molecule per se (eptinezumab, fremanezumab, galcanezumab) or a human antibody which binds to the N-terminals of CLR and RAMP1 (erenumab). They have all passed extensive clinical studies with significant effects and minor adverse events, subsequently they are now approved for migraine prophylaxis. While these molecules are effective in prophylaxis of chronic migraine their respective sites of action remains unclear.

\section{(i). Action on intracranial blood vessels.}

Dilatation of the middle meningeal arteries and neurogenic inflammation, in the dura mater, have been suggested as a triggering mechanism regarding migraine pathophysiology. The direct activation of trigeminal afferents in the dura causes a painful headache in humans; this observation was first made in 1940 via stimulation of these vascular nerves during surgery and extended more recently to include pia mater and small pial vessels (Fontaine et al., 2018). Similarities of the occurring headache with a migraine headache led to the vascular theory of migraine, which postulates that headaches is a disorder triggered by dilation of intra- or extra-cranial blood vessels. One aspect worth considering is that nearly all vasodilators given in a peripheral vein results in "migraine-like headache or migraine" (Ashina, Hansen, BO \& Olesen, 2017). However, this view is disputed since the same group of researchers did not observe that cerebral and meningeal arteries but primarily the extra-cranial arteries were dilated during a genuine migraine attack (Amin et al., 2013).

The first study of CGRP on intracranial arteries revealed very potent vasodilatation, independent of endothelial function and associated with activation of adenylyl cyclase in the vascular smooth cells (Edvinsson, Fredholm, Hamel, Jansen \& Verrecchia, 1985) and with a parallel reduction in intracellular calcium ion concentration (Erdling, Sheykhzade \& Edvinsson, 2017). Subsequent studies revealed that other members of the CGRP family of peptides also were vasodilators but had lower potency. While the perivascular sensory nerves had a rich supply of CGRP containing fibres, there is less expression of AMY and no ADM in nerve fibres on cerebral vessels. A subpopulation of CGRP positive TG neurons co-localized AMY, while no ADM-ir was seen (Edvinsson, Goadsby \& Uddman, 2001). The receptor components CLR and RAMP1-3 were demonstrated with qPCR, suggesting that AMY receptors might occur. In agreement human cerebral pial arteries, middle cerebral, superficial temporal and middle meningeal arteries have CLR, RAMP 1, RAMP2 and RAMP3 in the smooth muscle layers (Oliver, Wainwright, Edvinsson, Pickard \& Hill, 2002). The vascular endothelium contained CLR and RAMP2, but no RAMP1 or RAMP3. Functional study of 
cerebral arteries revealed strong vasodilator effects by CGRP and AMY (with or without endothelium) while only at very high concentration did $\mathrm{ADM}$ show a relaxant effect; $\mathrm{CGRP}_{8-37}$ blocked the relaxant response. Intracortical CGRP, AMY and ADM caused increased local cerebral blood flow by $42 \%, 32 \%$ and $15 \%$, respectively (Edvinsson, Goadsby \& Uddman, 2001). The responses to CGRP and AMY were blocked by CGRP $_{8-37}$, suggesting that the vasodilatation is mediated via CGRP receptors (both mRNA for CLR and RAMP1 were seen in the MCA) (Edvinsson, Goadsby \& Uddman, 2001). Studies of CT early revealed very low relaxant effect of brain vessels (Edvinsson, Ekman, Jansen, McCulloch \& Uddman, 1987); recent preliminary studies have not shown CT receptor-ir in the vessel walls, while the CGRP receptor components CLR and RAMP1 are present in human cerebral and middle meningeal arteries (Edvinsson et al., 2010). While gepants showed competitive inhibition (Edvinsson et al., 2010; Rubio-Beltran, Chan, Danser, MaassenVanDenBrink \& Edvinsson, 2020) the mAbs showed competitive antagonism with no depression of maximum CGRP induced relaxation in human middle meningeal and cerebral arteries (Ohlsson, Haanes, Kronvall, Xu, Snellman \& Edvinsson, 2019; Ohlsson, Kronvall, Stratton \& Edvinsson, 2018). The data supports a strong role for CGRP but the lack of AMY receptor component CTR, and few AM receptors are present their role is unlikely if induction of a migraine attack if vasodilatation is the key mechanism (Ashina, Hansen, BO \& Olesen, 2017; Walker et al., 2015). This view is in support by others who stated that the TGV system does not require a peripheral sensory input to be activated (Goadsby \& Akerman, 2012).

\section{(ii). Relation to the blood-brain barrier.}

In light of a vascular mechanisms of anti-migraine drugs, the possible relation of CGRP or its antagonists and the effect on dilation of cerebral arteries, must be considered. Importantly, the endothelium in these vessels restricts passage of molecules from the vessel lumen to the smooth muscle layers of the vascular wall containing CGRP nerve endings. The hypothesis was tested in isolated rat middle cerebral arteries that were cannulated and luminally perfused, enabling application of drugs to either the endothelium exposed in the lumen or the smooth muscle layers on the abluminal side of the artery (Edvinsson, Nilsson \& JansenOlesen, 2007). In these experiments, CGRP as well as AM, AMY and CT were relaxing the artery only when applied to the abluminal surface of the cerebral artery (Edvinsson, Nilsson \& Jansen-Olesen, 2007). Moreover, neither CGRP receptor antagonists (olcegepant and telcagepant) nor anti-CGRP antibodies blocked CGRPmediated dilation when applied to the lumen; they were effective only when applied to the abluminal side. On this basis, circulating CGRP receptor antagonists and mAbs against CGRP and the CGRP receptor, all of which are effective in migraine, do not seem to be able to cross the endothelial barrier to access targets in the brain vasculature.

A neurogenic inflammation occurring in the dura has also been proposed as trigger of migraine attacks (Pietrobon \& Moskowitz, 2013). However, numerous drugs that block the plasma protein extravasation component of neurogenic inflammation in the dura of animals have been tested in clinical trials but have not exhibited anti-migraine efficacy. Moreover, CGRP does not induce neurogenic inflammation in humans or rodents but mediates only the vasodilatory aspect of inflammation (Levy, Burstein \& Strassman, 2005; Schain, Melo-Carrillo, Stratton, Strassman \& Burstein, 2019). Although the idea of neurogenic inflammation has been discussed at length over the years the role in migraine is still not clear (Edvinsson, Haanes \& Warfvinge, 2019). Aspects for and against this hypothesis are still being discussed (Hadjikhani et al., 2020; Khan et al., 2019).

Considering the BBB, the question remains "where do the gepants and $\mathrm{mAbs}$ act" to relieve migraine headache. Looking at the dura mater, the cerebral circulation, the brain and the TG after administration of tracers like Evans Blue or radioactive compounds to calculate the permeability surface (PS product) passage showed that the dura and the TG were freely accessible (Eftekhari, Salvatore, Johansson, Chen, Zeng \& Edvinsson, 2015; Lundblad, Haanes, Grande \& Edvinsson, 2015). Induction of neurogenic inflammation using the Freud's Adjuvant or the "inflammation soup" revealed activation within the TG but no quantitative increase in PS product into the CNS. Subsequent measurements revealed minor passage by gepants $(2 \%)$ or none of the mAbs $(<0,01 \%)$ (Johnson, Morin, Wroblewski \& Johnson, 2019). This is in support of a more recent study by Noseda showing that labelled fremanezumab was distributed to cranial sensory and 
autonomic ganglia and to the dura mater but not to the CNS in rats with uncompromised blood-brain barrier (Noseda et al., 2020). Since the TG and its peripheral ramifications (TGV system) are without the protection of the blood-brain barrier it is likely that the antimigraine site of action of the CGRP group of anti-migraine drugs reside here. This is also the reason why there is few CNS related side-effects.

(iii). Action on neuron cell bodies and nociceptive fibres.

The trigeminal system provides the link between the peripheral primary afferents and the central terminals of the trigeminal nucleus caudalis (TNC). Activation of this pathway may result in sensitization within secondorder neurons and drive the CNS aspects of the migraine attacks (May \& Burstein, 2019). Secondly, it has been demonstrated that activation of hypothalamus is early (prodromal) site where the migraine attack starts (May \& Burstein, 2019; Schulte, Mehnert \& May, 2020; Schulte, Menz, Haaker \& May, 2020). Connectivity studies have revealed that other CNS regions are subsequently activated including the brainstem from with links are available to activate or modulate the trigeminal system function (May \& Burstein, 2019). In both hypothesis the trigeminal system plays a key role and currently available mAbs towards CGRP and the CGRP receptor are effective (Edvinsson, Haanes, Warfvinge \& Krause, 2018) despite their inability to penetrate the blood-brain barrier (Lundblad, Haanes, Grande \& Edvinsson, 2015; Noseda et al., 2020).

Each of the peptides of the CGRP family exhibits a distinct selection of biological actions (Poyner et al., 2002). CGRP and AMY are the most closely related peptides in terms of amino acid sequence, which may cause an overlap in their ability to activate their receptors. CGRP and AMY are reported to have effects related to pain, though there are still limited data and it is unclear how much overlap there is because the peptides are usually not studied simultaneously. The relative potency of CGRP, CT, AMY and AM at the different receptor complexes is complicated and challenging because of cross-reactivity (Hay, Garelja, Poyner \& Walker, 2018; Hendrikse, Bower, Hay \& Walker, 2018). In addition, release of a peptide will result in a very high concentration just at the receptor site, while circulating levels vary considerably. The study of the nodes of Ranvier in the trigeminal system demonstrated CGRP containing boutons with CGRP containing vesicles in C-fibres that may directly release CGRP to reach the adjacent Ao-fibres with CGRP receptors (Edvinsson et al., 2019).

The possibility of intercellular cross-talk in the TG was recently proposed to result in a feedback loop sensitizing neurons (Messlinger, Balcziak \& Russo, 2020). Apart from direct gap-junction communication between SGC's and neurons, paracrine signalling may also occur. CGRP locally released by neurons or Cfibres could potentially activate receptors located on SGC's, other neurons or auto receptors. This activation may in turn affect gene expression, neurotropic factors, neuropeptides or receptor regulations.

There exists few direct functional studies on the various cells in the TG. Hypothetically, a range of plausible targets, available to circulating drugs with low BBB permeability, can be noted: (i) Peripheral terminals of nociceptive A $\delta$-fibers and C-fibres innervating various cranial structures, (ii) Receptors expressed at or proximal to the nodes of Ranvier on Aס-fibers (Edvinsson et al., 2019), (iii) Neuron cell bodies (notably, in the TG these are vigorously enveloped by SGCs which may act as a gate-keeper to systemically introduced drugs) and (iv) the SGCs and Schwann cells in the TG (likely a complex signalling relationship between these cells and proximal neurons/axons (Messlinger, Balcziak \& Russo, 2020).

A recent study demonstrated that the CGRP receptor antagonist Erenumab was internalized by both the CGRP receptor and AMY1 receptor in cultured TG neurons (Bhakta, Vuong, Taura, Wilson, Stratton \& Mackenzie, 2021). Thus, there is a possibility of paracrine CGRP signalling occurring with SGC's expressing either the CGRP receptor or the AMY1 receptor. Both receptor activation pathways lead to an upregulation of cAMP which in turn could further sensitize neurons and axons to noxious stimuli.

Non-myelinating Schwann cells form Remak bundles around parts of C-fibre axons. They are vital in providing trophic factors and regenerating damaged C-fibres (Murinson \& Griffin, 2004). Disruption of non-myelinating Schwann cell ErbB signalling in mice resulted in a progressive sensory loss. This suggests intercellular signalling between non-myelinating Schwann cells and unmyelinated sensory fibres and is critical for C-fibre and Schwann cell survival. The implications of SGC and Schwann cell interactions with neurons is a field 
of research not yet fully understood. In part due to difficulties in studying the structures in vitro without disrupting the neuron-glia architecture.

(iv). Action in the nodes of Ranvier - axon-axon interaction.

The exploration of CGRP and its receptor have so far yielded successful scientific breakthroughs and therapies. RAMP1 and CLR are co-expressed mainly in larger neurons and in the thinly myelinated fibres, typically identified as Aס-fibres (Edvinsson et al., 2019; Eftekhari, Salvatore, Calamari, Kane, Tajti \& Edvinsson, 2010). CGRP receptors are found distally in the dura mater, associated both with the vessels and at avascular sites. In larger arteries, these fibres run in the adventitia, close to the C-fibres. Interestingly, the C-fibres storing CGRP could align with adjacent nodes of Ranvier in the Aס-fibres (Edvinsson et al., 2019). This led to the suggestion that at the nodes of Ranvier local release of CGRP may act on CGRP receptors on the A $\delta$-fibres to modify the sensory signalling. In this region, CLR/RAMP1 as well as a complete CGRP receptor antibody immunoreactivity were observed on the A $\delta$-fibres and associated with protein kinase A, which provides a link towards the possible phosphorylation of ion channels in Aó-fibres (Edvinsson et al., 2019). This mechanism may alter ion channel activity at the A $\delta$-fibres and contribute to the understanding of pain signalling and perhaps also the sensitization processes. The nodes are defined by their lack of the many layers of myelinated Schwann cells, which is why they may be a target for therapeutic drugs such as gepants and mAbs to interact within the trigeminal system and the pain perception.

\section{FUTURE PERSPECTIVES.}

Future research should aim to compare quantitatively the expression of the discussed peptides and receptors, both in relation to sex and age. Preliminary expression studies suggest that (i) CGRP and CT are the most expressed of the studied peptides, (ii) that CGRP receptor and CTR are the most frequently expressed receptor types and (iii) that $\mathrm{AM}_{2}, \mathrm{AMY}_{1}$ and $\mathrm{AMY}_{3}$ receptors occur in rat TG, but are mainly localized to the SGCs. The demonstration of the specific ligands and receptor sites in TG neurons highlights the importance of CGRP and the CGRP receptor as viable contributors in primary headache disorders. Future investigations of the expression of the CGRP-family of peptides near the node of Ranvier is warranted. Understanding the location and distribution is important in deciding on how to identify future targets for anti-migraine medications and this will facilitate future drug development and ultimately enrich the therapeutic options for the patients.

\section{Figure Legends.}

Figure 1. Immunohistochemical demonstration of localization of CGRP, CT, Amylin and Adrenomedullin in the trigeminal ganglion.A. CGRP is expressed in a granular pattern in many neurons, mainly in small- to medium-sized neurons (arrow heads). The cellular CGRP is expressed in vesicles (insert). In addition, pearllike CGRP immunoreactivity was detected in fibers that are of the C-type of sensory unmyelinated nerves (long arrows). The myelinated fibers do not contain CGRP. Short arrow points at a large negative neuron. B . CT immunoreactivity displayed a similar pattern as for CGRP; granular staining of small- to mediumsized neurons (arrow heads, insert) and pearl-like staining of fibers. Also, SGCs were CT immunoreactive (asterisks). Arrows point at a large negative neuron. C . AMY was exclusively expressed in the neurons, mainly small to medium sized (arrow heads). In some of the cells, the expression was granular, but in others, a general cytoplasmic immunoreactivity. Arrows point at a large negative neuron. Insert shows three different cells: one negative and two amylin immunoreactive. D . AM was expressed in the glial cells, both the SGCs (arrow heads) and cells enveloping the neuronal processes (arrows), probably myelinating cells.

Figure 2. Schematic illustration of the differential distribution of the CGRP family of peptides and receptors. The CGRP receptor (CLR/RAMP1) is mainly expressed in the large neurons and SGCs, the Adrenomedullin $_{2}$ receptor (CLR/RAMP3) in some SGCs, the Amylin ${ }_{1}$ receptor (CTR/RAMP1) in large neurons and SGCs, and the Amylin 3 receptor (CTR/RAMP3) in some SGCs Modified from Edvinsson, Grell \& Warfvinge, 2020.

\section{REFERENCES}


(2018). Global, regional, and national incidence, prevalence, and years lived with disability for 354 diseases and injuries for 195 countries and territories, 1990-2017: a systematic analysis for the Global Burden of Disease Study 2017. Lancet (London, England) 392: 1789-1858.

Amin FM, Asghar MS, Hougaard A, Hansen AE, Larsen VA, de Koning PJ, et al. (2013). Magnetic resonance angiography of intracranial and extracranial arteries in patients with spontaneous migraine without aura: a cross-sectional study. Lancet Neurol 12: 454-461.

Amrutkar DV, Ploug KB, Hay-Schmidt A, Porreca F, Olesen J, \& Jansen-Olesen I (2012). mRNA expression of 5-hydroxytryptamine $1 \mathrm{~B}, 1 \mathrm{D}$, and $1 \mathrm{~F}$ receptors and their role in controlling the release of calcitonin generelated peptide in the rat trigeminovascular system. Pain 153: 830-838.

Ashina M, Hansen JM, BO AD, \& Olesen J (2017). Human models of migraine - short-term pain for long-term gain. Nat Rev Neurol 13:713-724.

Ashina MD, D. Bonner, JH et al (2019) A phase 2, randomized, double-blind, placebo-controlled study to evaluate the efficacy and safety of $A M G 301$ in migraine prevention. . vol. Athens.

Bhakta M, Vuong T, Taura T, Wilson DS, Stratton JR, \& Mackenzie KD (2021). Migraine therapeutics differentially modulate the CGRP pathway. Cephalalgia: 0333102420983282.

Bower RL, Eftekhari S, Waldvogel HJ, Faull RL, Tajti J, Edvinsson L, et al. (2016). Mapping the calcitonin receptor in human brain stem. Am J Physiol Regul Integr Comp Physiol 310: R788-793.

Charles A, \& Pozo-Rosich P (2019). Targeting calcitonin gene-related peptide: a new era in migraine therapy. Lancet (London, England) 394: 1765-1774.

Cooper GJ, Willis AC, Clark A, Turner RC, Sim RB, \& Reid KB (1987). Purification and characterization of a peptide from amyloid-rich pancreases of type 2 diabetic patients. Proc Natl Acad Sci U S A 84: 8628-8632.

Edvinsson JCA, Warfvinge K, Krause DN, Blixt FW, Sheykhzade M, Edvinsson L, et al. (2019). Cfibers may modulate adjacent Adelta-fibers through axon-axon CGRP signaling at nodes of Ranvier in the trigeminal system. J Headache Pain 20: 105.

Edvinsson L (2011). Tracing neural connections to pain pathways with relevance to primary headaches. Cephalalgia 31: 737-747.

Edvinsson L, Alm R, Shaw D, Rutledge RZ, Koblan KS, Longmore J, et al. (2002). Effect of the CGRP receptor antagonist BIBN4096BS in human cerebral, coronary and omental arteries and in SK-N-MC cells. Eur J Pharmacol 434: 49-53.

Edvinsson L, Chan KY, Eftekhari S, Nilsson E, de Vries R, Saveland H, et al. (2010). Effect of the calcitonin gene-related peptide (CGRP) receptor antagonist telcagepant in human cranial arteries. Cephalalgia 30: 1233-1240.

Edvinsson L, Ekman R, Jansen I, McCulloch J, \& Uddman R (1987). Calcitonin gene-related peptide and cerebral blood vessels: distribution and vasomotor effects. J Cereb Blood Flow Metab 7: 720-728.

Edvinsson L, Fredholm BB, Hamel E, Jansen I, \& Verrecchia C (1985). Perivascular peptides relax cerebral arteries concomitant with stimulation of cyclic adenosine monophosphate accumulation or release of an endothelium-derived relaxing factor in the cat. Neurosci Lett 58: 213-217.

Edvinsson L, Goadsby PJ, \& Uddman R (2001). Amylin: localization, effects on cerebral arteries and on local cerebral blood flow in the cat. ScientificWorldJournal 1: 168-180.

Edvinsson L, Grell AS, \& Warfvinge K (2020). Expression of the CGRP Family of Neuropeptides and their Receptors in the Trigeminal Ganglion. J Mol Neurosci 70: 930-944.

Edvinsson L, Haanes KA, \& Warfvinge K (2019). Does inflammation have a role in migraine? Nat Rev Neurol 15: 483-490. 
Edvinsson L, Haanes KA, Warfvinge K, \& Krause DN (2018). CGRP as the target of new migraine therapies - successful translation from bench to clinic. Nat Rev Neurol 14: 338-350.

Edvinsson L, Nilsson E, \& Jansen-Olesen I (2007). Inhibitory effect of BIBN4096BS, CGRP(8-37), a CGRP antibody and an RNA-Spiegelmer on CGRP induced vasodilatation in the perfused and non-perfused rat middle cerebral artery. Br J Pharmacol 150: 633-640.

Eftekhari S, Gaspar RC, Roberts R, Chen TB, Zeng Z, Villarreal S, et al. (2016). Localization of CGRP receptor components and receptor binding sites in rhesus monkey brainstem: A detailed study using in situ hybridization, immunofluorescence, and autoradiography. J Comp Neurol 524: 90-118.

Eftekhari S, Salvatore CA, Calamari A, Kane SA, Tajti J, \& Edvinsson L (2010). Differential distribution of calcitonin gene-related peptide and its receptor components in the human trigeminal ganglion. Neuroscience 169: $683-696$.

Eftekhari S, Salvatore CA, Johansson S, Chen TB, Zeng Z, \& Edvinsson L (2015). Localization of CGRP, CGRP receptor, PACAP and glutamate in trigeminal ganglion. Relation to the blood-brain barrier. Brain Res 1600: 93-109.

Eftekhari S, Warfvinge K, Blixt FW, \& Edvinsson L (2013). Differentiation of nerve fibers storing CGRP and CGRP receptors in the peripheral trigeminovascular system. J Pain 14: 1289-1303.

Erdling A, Sheykhzade M, \& Edvinsson L (2017). Differential inhibitory response to telcagepant on alphaCGRP induced vasorelaxation and intracellular $\mathrm{Ca} 2+$ levels in the perfused and non-perfused isolated rat middle cerebral artery. J Headache Pain 18: 61.

Ferrero H, Larrayoz IM, Gil-Bea FJ, Martinez A, \& Ramirez MJ (2018). Adrenomedullin, a Novel Target for Neurodegenerative Diseases. Mol Neurobiol.

Ferrero H, Larrayoz IM, Martisova E, Solas M, Howlett DR, Francis PT, et al. (2018). Increased Levels of Brain Adrenomedullin in the Neuropathology of Alzheimer's Disease. Mol Neurobiol 55:5177-5183.

Findlay DM, \& Sexton PM (2004). Calcitonin. Growth Factors 22:217-224.

Fontaine D, Almairac F, Santucci S, Fernandez C, Dallel R, Pallud J, et al. (2018). Dural and pial painsensitive structures in humans: new inputs from awake craniotomies. Brain 141:1040-1048.

GBD 2016 Disease and Injury Incidence and Prevalence Collaborators (2017). Global, regional, and national incidence, prevalence, and years lived with disability for 328 diseases and injuries for 195 countries, 19902016: a systematic analysis for the Global Burden of Disease Study 2016. Lancet (London, England) 390: 1211-1259.

Gingell JJ, Hendrikse ER, \& Hay DL (2019). New Insights into the Regulation of CGRP-Family Receptors. Trends Pharmacol Sci 40:71-83.

Goadsby PJ, \& Akerman S (2012). The trigeminovascular system does not require a peripheral sensory input to be activated-migraine is a central disorder. Focus on 'Effect of cortical spreading depression on basal and evoked traffic in the trigeminovascular sensory system'. Cephalalgia 32: 3-5.

Goadsby PJ, \& Edvinsson L (1994). Joint 1994 Wolff Award Presentation. Peripheral and central trigeminovascular activation in cat is blocked by the serotonin (5HT)-1D receptor agonist 311C90. Headache 34:394-399.

Goadsby PJ, Edvinsson L, \& Ekman R (1988). Release of vasoactive peptides in the extracerebral circulation of humans and the cat during activation of the trigeminovascular system. Ann Neurol 23:193-196.

Goadsby PJ, Holland PR, Martins-Oliveira M, Hoffmann J, Schankin C, \& Akerman S (2017). Pathophysiology of Migraine: A Disorder of Sensory Processing. Physiol Rev 97: 553-622. 
Goadsby PJ, \& Knight YE (1997). Direct evidence for central sites of action of zolmitriptan (311C90): an autoradiographic study in cat. Cephalalgia 17: 153-158.

Hadjikhani N, Albrecht DS, Mainero C, Ichijo E, Ward N, Granziera C, et al. (2020). Extra-Axial Inflammatory Signal in Parameninges in Migraine with Visual Aura. Ann Neurol 87: 939-949.

Hay DL (2017). Amylin. Headache 57 Suppl 2: 89-96.

Hay DL, Garelja ML, Poyner DR, \& Walker CS (2018). Update on the pharmacology of calcitonin/CGRP family of peptides: IUPHAR Review 25. Br J Pharmacol 175: 3-17.

Hay DL, Harris PW, Kowalczyk R, Brimble MA, Rathbone DL, Barwell J, et al. (2014). Structure-activity relationships of the N-terminus of calcitonin gene-related peptide: key roles of alanine- 5 and threonine- 6 in receptor activation. Br J Pharmacol 171:415-426.

Hay DL, \& Pioszak AA (2016). Receptor Activity-Modifying Proteins (RAMPs): New Insights and Roles. Annu Rev Pharmacol Toxicol 56:469-487.

Hendrikse ER, Bower RL, Hay DL, \& Walker CS (2018). Molecular studies of CGRP and the CGRP family of peptides in the central nervous system. Cephalalgia: 333102418765787.

Ho TW, Edvinsson L, \& Goadsby PJ (2010). CGRP and its receptors provide new insights into migraine pathophysiology. Nat Rev Neurol 6:573-582.

Hoffmann J, Wecker S, Neeb L, Dirnagl U, \& Reuter U (2012). Primary trigeminal afferents are the main source for stimulus-induced CGRP release into jugular vein blood and CSF. Cephalalgia 32:659-667.

Irimia P, Martínez-Valbuena I, Mínguez-Olaondo A, Domínguez-Vivero C, Sánchez-Arias J-A, MartínezVila E, et al. Interictal amylin levels in chronic migraine patients: A case-control study. Cephalalgia 0: 0333102420977106.

Johnson KW, Morin SM, Wroblewski VJ, \& Johnson MP (2019). Peripheral and central nervous system distribution of the CGRP neutralizing antibody [(125)I] galcanezumab in male rats. Cephalalgia 39: 12411248 .

Juhl L, Petersen KA, Larsen EH, Jansen-Olesen I, \& Olesen J (2006). The in vivo effect of adrenomedullin on rat dural and pial arteries. Eur J Pharmacol 538: 101-107.

Khan S, Amin FM, Fliedner FP, Christensen CE, Tolnai D, Younis S, et al. (2019). Investigating macrophagemediated inflammation in migraine using ultrasmall superparamagnetic iron oxide-enhanced 3T magnetic resonance imaging. Cephalalgia 39: 1407-1420.

Kitamura K, Kangawa K, Kawamoto M, Ichiki Y, Nakamura S, Matsuo H, et al. (1993). Adrenomedullin: a novel hypotensive peptide isolated from human pheochromocytoma. Biochem Biophys Res Commun 192:553560 .

Labastida-Ramirez A, Rubio-Beltran E, Haanes KA, Chan KY, Garrelds IM, Johnson KW, et al. (2020). Lasmiditan inhibits calcitonin gene-related peptide release in the rodent trigeminovascular system. Pain 161: 1092-1099.

Lennerz JK, Ruhle V, Ceppa EP, Neuhuber WL, Bunnett NW, Grady EF, et al. (2008). Calcitonin receptorlike receptor (CLR), receptor activity-modifying protein 1 (RAMP1), and calcitonin gene-related peptide (CGRP) immunoreactivity in the rat trigeminovascular system: differences between peripheral and central CGRP receptor distribution. J Comp Neurol 507: 1277-1299.

Levy D, Burstein R, \& Strassman AM (2005). Calcitonin gene-related peptide does not excite or sensitize meningeal nociceptors: implications for the pathophysiology of migraine. Ann Neurol 58: 698-705. 
Lundblad C, Haanes KA, Grande G, \& Edvinsson L (2015). Experimental inflammation following dural application of complete Freund's adjuvant or inflammatory soup does not alter brain and trigeminal microvascular passage. J Headache Pain 16: 91.

May A (2017). Understanding migraine as a cycling brain syndrome: reviewing the evidence from functional imaging. Neurol Sci 38:125-130.

May A, \& Burstein R (2019). Hypothalamic regulation of headache and migraine. Cephalalgia: 333102419867280 .

McLatchie LM, Fraser NJ, Main MJ, Wise A, Brown J, Thompson N, et al. (1998). RAMPs regulate the transport and ligand specificity of the calcitonin-receptor-like receptor. Nature 393: 333-339.

Messlinger K, Balcziak LK, \& Russo AF (2020). Cross-talk signaling in the trigeminal ganglion: role of neuropeptides and other mediators. Journal of Neural Transmission 127: 431-444.

Messlinger K, \& Russo AF (2019). Current understanding of trigeminal ganglion structure and function in headache. Cephalalgia 39:1661-1674.

Mietlicki-Baase EG, McGrath LE, Koch-Laskowski K, Krawczyk J, Reiner DJ, Pham T, et al. (2017). Amylin receptor activation in the ventral tegmental area reduces motivated ingestive behavior. Neuropharmacology 123: $67-79$.

Miller S, Liu H, Warfvinge K, Shi L, Dovlatyan M, Xu C, et al.(2016). Immunohistochemical localization of the calcitonin gene-related peptide binding site in the primate trigeminovascular system using functional antagonist antibodies. Neuroscience 328: 165-183.

Mulder IA, Li M, de Vries T, Qin T, Yanagisawa T, Sugimoto K, et al. (2020). Anti-migraine Calcitonin Gene-Related Peptide Receptor Antagonists Worsen Cerebral Ischemic Outcome in Mice. Ann Neurol 88: 771-784.

Murinson BB, \& Griffin JW (2004). C-fiber structure varies with location in peripheral nerve. Journal of Neuropathology \& Experimental Neurology 63: 246-254.

Noseda R, Schain AJ, Melo-Carrillo A, Tien J, Stratton J, Mai F, et al. (2020). Fluorescently-labeled fremanezumab is distributed to sensory and autonomic ganglia and the dura but not to the brain of rats with uncompromised blood brain barrier. Cephalalgia 40:229-240.

Ohlsson L, Haanes KA, Kronvall E, Xu C, Snellman J, \& Edvinsson L (2019). Erenumab (AMG 334), a monoclonal antagonist antibody against the canonical CGRP receptor, does not impair vasodilatory or contractile responses to other vasoactive agents in human isolated cranial arteries. Cephalalgia 39: 1745-1752.

Ohlsson L, Kronvall E, Stratton J, \& Edvinsson L (2018). Fremanezumab blocks CGRP induced dilatation in human cerebral, middle meningeal and abdominal arteries. J Headache Pain 19: 66.

Oliver KR, Wainwright A, Edvinsson L, Pickard JD, \& Hill RG (2002). Immunohistochemical localization of calcitonin receptor-like receptor and receptor activity-modifying proteins in the human cerebral vasculature. J Cereb Blood Flow Metab 22: 620-629.

Pietrobon D, \& Moskowitz MA (2013). Pathophysiology of migraine. Annu Rev Physiol 75: 365-391.

Poyner DR, Sexton PM, Marshall I, Smith DM, Quirion R, Born W, et al. (2002). International Union of Pharmacology. XXXII. The mammalian calcitonin gene-related peptides, adrenomedullin, amylin, and calcitonin receptors. Pharmacol Rev 54: 233-246.

Rubio-Beltran E, Chan KY, Danser AJ, MaassenVanDenBrink A, \& Edvinsson L (2020). Characterisation of the calcitonin gene-related peptide receptor antagonists ubrogepant and atogepant in human isolated coronary, cerebral and middle meningeal arteries. Cephalalgia 40: 357-366. 
Satoh F, Takahashi K, Murakami O, Totsune K, Sone M, Ohneda M, et al. (1996). Immunocytochemical localization of adrenomedullin-like immunoreactivity in the human hypothalamus and the adrenal gland. Neurosci Lett 203: 207-210.

Saxena PR, \& Den Boer MO (1991). Pharmacology of antimigraine drugs. J Neurol 238 Suppl 1: S28-35.

Schain AJ, Melo-Carrillo A, Stratton J, Strassman AM, \& Burstein R (2019). CSD-Induced Arterial Dilatation and Plasma Protein Extravasation Are Unaffected by Fremanezumab: Implications for CGRP's Role in Migraine with Aura. J Neurosci 39: 6001-6011.

Schulte LH, \& May A (2017). Of generators, networks and migraine attacks. Curr Opin Neurol 30: 241-245.

Schulte LH, Mehnert J, \& May A (2020). Longitudinal Neuroimaging over 30 Days: Temporal Characteristics of Migraine. Ann Neurol 87:646-651.

Schulte LH, Menz MM, Haaker J, \& May A (2020). The migraineur's brain networks: Continuous resting state fMRI over 30 days. Cephalalgia 40: 1614-1621.

Schultz N, Byman E, Fex M, \& Wennstrom M (2017). Amylin alters human brain pericyte viability and NG2 expression. J Cereb Blood Flow Metab 37: 1470-1482.

Serrano J, Uttenthal LO, Martinez A, Fernandez AP, Martinez de Velasco J, Alonso D, et al. (2000). Distribution of adrenomedullin-like immunoreactivity in the rat central nervous system by light and electron microscopy. Brain Res 853: 245-268.

Walker CS, Eftekhari S, Bower RL, Wilderman A, Insel PA, Edvinsson L, et al. (2015). A second trigeminal CGRP receptor: function and expression of the AMY1 receptor. Ann Clin Transl Neurol 2:595-608.
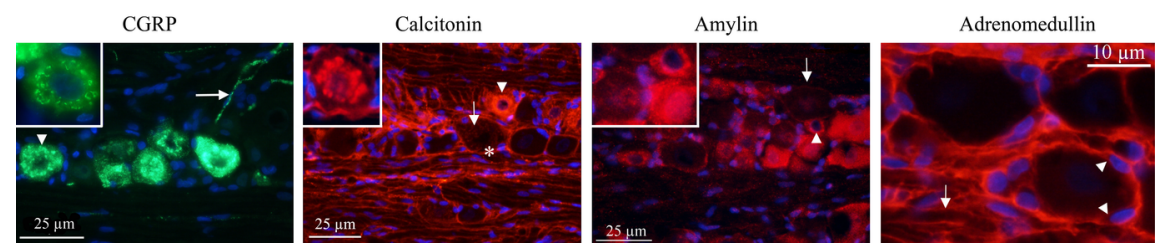

CGRP receptor

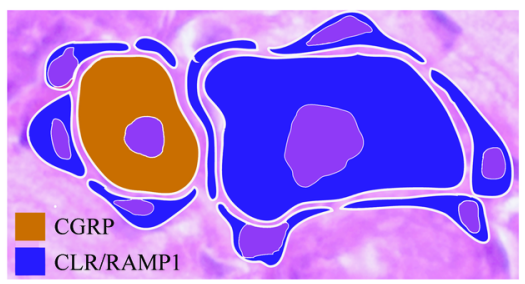

Amylin $_{1}$ receptor

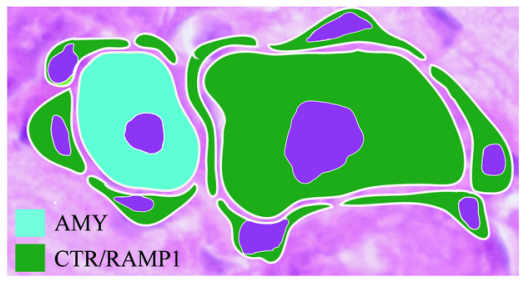

Adrenomedullin $_{2}$ receptor

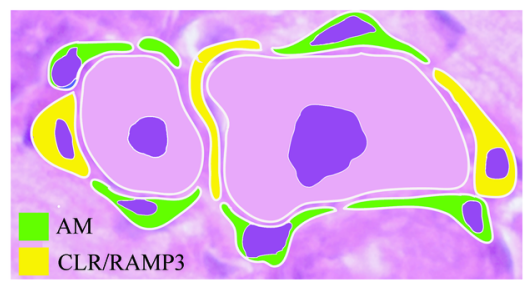

Amylin $_{3}$ receptor

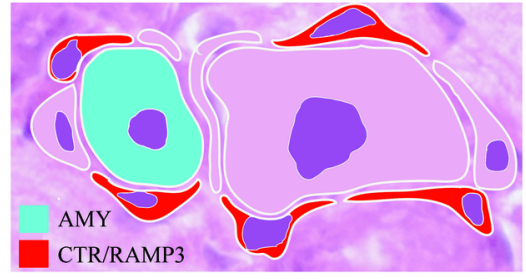

